\title{
Scolia
}

Revue de linguistique

$30 \mid 2016$

Des connecteurs argumentatifs aux opérateurs discursifs

\section{À peine, opérateur discursif}

Jesús Vázquez Molina

\section{(2) OpenEdition}

Journals

Édition électronique

URL : http://journals.openedition.org/scolia/504

DOI : $10.4000 /$ scolia. 504

ISSN : 2677-4224

Éditeur

Presses universitaires de Strasbourg

\section{Édition imprimée}

Date de publication : 19 juin 2016

Pagination : 105-121

ISBN : 978-2-86820-944-3

ISSN : 1253-9708

Référence électronique

Jesús Vázquez Molina, «À peine, opérateur discursif », Scolia [En ligne], 30 | 2016, mis en ligne le 09 avril 2019, consulté le 19 mai 2019. URL : http://journals.openedition.org/scolia/504 ; DOI : 10.4000/ scolia. 504 


\title{
À peine, opérateur discursif
}

\author{
Jesús VÁZqueZ MolinA \\ Université d'Oviedo, \\ groupe OPÉRAS \\ vazquezjesus@uniovi.es
}

\section{Introduction}

La multiplicité fonctionnelle semble être l'une des caractéristiques de la locution adverbiale à peine, car elle est toujours présentée comme polysémique dans les dictionnaires, les grammaires, et les travaux linguistiques en général.

Nous allons procéder d'une manière différente en comparaison avec la plupart de ces travaux, évitant les a priori contenus dans les études grammaticales, et analysant cet élément d'une manière globale, en tant qu'opérateur discursif. Pour l'analyse détaillée de son comportement, nous travaillerons sur des exemples tirés de discours divers, oraux et écrits, ces derniers appartenant à la presse ou à la littérature contemporaine, et cela afin de pouvoir analyser le comportement de $a$ peine dans toute sa diversité d'emplois. Enfin, l'inventaire d'une série de propriétés formelles et morphosyntaxiques nous permettra d'établir des critères efficaces pour déterminer les significations possibles de cet opérateur. Plus concrètement, notre but sera de vérifier si l'entité lexicale à peine correspond à une ou à plusieurs entités sémantiques. 


\section{1. À peine dans la grammaire et dans la linguistique}

Les grammaires et les dictionnaires se réfèrent à cette locution en lui attribuant deux ou trois significations différentes. Un à peine quantitatif et sémi-négatif, équivalent de presque pas, devant ou après un adjectif ou un verbe: Un texte à peine visible/On l'entend à peine / $A$ peine l'entend-on (Hanse \& Blampain, 2012: 457). Un deuxième sens, lui aussi quantitatif, est souvent cité, équivalent de tout au plus, seulement, quand cette unité précède un numéral ou un indéfini: Ça fait à peine dix euros (P. Rob., 2011: 1842). Il faut remarquer que dans ses significations quantitatives, à peine est rangé parmi les adverbes exprimant le degré faible, à côté de peu et un peu (Grevisse \& Goosse, 2011). Sandfeld (1977) ajoute que à peine peut être modifié par c'est et porter sur une proposition introduite par si. Cette phrase a la valeur d'une complétive, et elle peut être simplifiée par la suppression de c'est: C'est à peine si je savais où la trouver. À peine s'il m'avait regardée (Sandfeld, 1977: 362). Enfin, on décrit aussi un sens temporel ou aspectuel (à l'instant, tout juste): Nous commençons à peine à travailler. (Dict. Acad. Fr., s.v. peine). Dans ce dernier usage à peine est fréquemment dans une proposition pour marquer le peu de temps qui sépare deux actions, en corrélation ou non avec que pour introduire la subordonnée: À peine dans un endroit, il se transportait dans un autre (Grevisse \& Goosse, 2011: 1552). J'étais à peine sorti qu'il commença à pleuvoir (P. Rob., 2011: 1842).

Pour ce qui est des études linguistiques qui s'occupent de cet élément, la plupart des linguistes s'accordent sur le caractère polysémique de à peine, notamment Bat-Zeev Shyldkrot (2010). Mais les analyses contrastives avec d'autres adverbes sont bien prédominantes: presque (Ducrot, 1972; Jayez, 1987; Rouanne, 1998), juste (Leeman, 2004; Mellet \& Monte, 2009), difficilement (Bat-Zeev Shyldkrot, 2012).

Ayant commenté ailleurs bien plus en détail ces études et leur utilité dans l'analyse de à peine ${ }^{1}$, on se limitera donc à quelques appréciations sur certaines idées récurrentes. Ainsi, Ducrot, comparant presque et à peine, conclut que ce qui fait la spécificité de à peine est que dans certains contextes, cet élément «devient un quasi synonyme de peu

1 Dans Vázquez Molina (à paraître). 
et dans d'autres se rapproche de seulement» (Ducrot, 1991:258)2 . Il évoque, en outre, son effet dévalorisant, particulièrement perçu dans certains contextes: Il gagne à peine mille francs par mois. Cet effet serait une fonction secondaire attachée à cette unité, un sous-entendu. Ce sous-entendu «minorant» est symétrique du sousentendu «majorant», lié à presque, qui pose «non loin de Y» (Ducrot, 1991: 265). L'orientation argumentative différente de presque et de à peine se manifeste également quand on examine le comportement des expressions au plus et au moins; au moins se comportant comme presque et au plus comme à peine (Leeman, 2004).

Mellet \& Monte (2009) montrent que, dans certains emplois, juste et à peine semblent pouvoir être substitués sans que le changement sémantique ne dépasse une simple nuance. Ils soulignent également que juste / à peine sont également commutables quand ils se combinent avec des expressions quantitatives. Pourtant, la présence de à peine oriente vers le moins et "permet des argumentations basées sur la faiblesse numérique des entités considérées» (Mellet \& Monte, 2009: 47).

Quant à Bat-Zeev Shyldkrot (2010, 2012), elle définit trois emplois de à peine (qui coïncident en gros avec ceux décrits dans les grammaires): quantifieur, à valeur de quasi-négation, restrictiflimitatif (accompagné d'un élément exprimant la quantité), et, enfin, temporel-aspectuel.

Ce bref aperçu nous laisse entrevoir, d'abord, la réitération des propriétés de à peine qui se répètent dans les travaux grammaticaux sans qu'il n'existe de critères solides pour y établir une ou plusieurs significations. Et d'autre part, le souci évident des sémanticiens de déceler les valeurs sémantiques renfermées dans la polysémie de ce marqueur qui suscitent quelques remarques utiles à sa description, notamment en le comparant avec d'autres éléments "quasi-synonymes " dans certains contextes, afin d'en affiner la description. Pourtant, à notre avis, ces analyses n'atteignent pas l'objectif de déterminer une certaine valeur commune aux différents emplois de à peine, et les critères appliqués sont fort divers et pas toujours vérifiables, si bien qu'il reste encore à faire une description complète qui inclue, par

2 On va retrouver la même idée chez Bat-Zeev Shyldkrot (2010: 13): emplois de «quasi-négation», et « restrictif-limitatif», respectivement. 
exemple, les valeurs dites «temporelles» de la locution, qui n'ont pas été analysées en profondeur.

\section{Pour une analyse sémantico-pragmatique}

Notre élément est considéré comme un marqueur, ou plus précisément comme un opérateur discursif. Par opérateur, on fait référence à la fonction d'instruction des opérations sémanticopragmatiques qui permettent d'interpréter le sens dans le discours ${ }^{3}$.

L'examen, même superficiel, des exemples suivants, permet d'affirmer qu'il y a des différences importantes entre ces occurrences de à peine (Ap): d'abord, entre (1-3), d'un côté, et (4-5) de l'autre. En effet, dans les exemples (1), (2) et (3), Ap semble fonctionner comme un adverbe de constituant, ce qui n'est pas le cas pour (4) et (5), où il connecte deux propositions:

1) Elle quitta le vestibule sur un au revoir marmonné, à peine audible. (Lemaitre, Au revoir là-haut, 2013)

2) Pas une glissade pour faire le show sur le court Arthur Ashe. À peine une petite colère passagère pour une sombre histoire de challenge non favorable. On n'a pas reconnu Gaël Monfils lors de sa victoire face à Grigor Dimitrov en 8es à l'US Open, mardi. (20 minutes, 04/09/2014)

3) Mais cette histoire ne finit pas, elle commence à peine. (Nothomb, Hygiène de l'assassin, 2004)

4) À peine la frégate amarrée, le sculpteur a sauté à terre. (Énard, Parle-leur de batailles, de rois et d'éléphants, 2010)

5) À peine avait-il fait deux mètres que Charles le rappela. (Foenkinos, La délicatesse, 2009)

On partira donc de l'hypothèse que $A p$ est certes un élément polysémique, quoiqu'on puisse, en parallèle, déterminer une certaine valeur sémantique commune qui distinguerait cet opérateur d'autres qui lui sont proches. On affirmera donc que l'entité lexicale $(A p)$ correspond au moins à deux entités sémantiques $A p_{1}$ et $A p_{2}$, qui peuvent être distinguées selon leurs propriétés distributionnelles et sémantiques.

3 Voir notamment l'Introduction du volume d'Anscombre et al. (2013), écrite par Donaire. 


\section{1. $A p_{1}$}

\subsubsection{Propriétés distributionnelles}

a) Dès le niveau le plus superficiel, on peut distinguer deux comportements formels qui pourraient confirmer l'hypothèse de l'existence de deux entités sémantiques, $A p_{1}$ et $A p_{2}$. La première répond à la structure canonique à peine $X, A p_{1}$ fonctionnant comme un adverbe de constituant qui étend sa portée vers $X$ (exemples 1-3), tandis que la structure de $A p_{2}$ répond généralement au type à peine $X$, (que) $Y$ (exemples 4-5). Ils diffèrent également quant à la nature de $X$, formée, dans le cas de $A p_{1}$, le plus souvent d'un adjectif ou un participe passé, tandis qu'en (4) et (5), comme on peut le vérifier, $X$ et $Y$ sont des phrases et non des constituants phrastiques.

b) Mais $A p_{1}$ peut également être sous la dépendance d'un verbe (exemple 3), et même d'un substantif, ce qui n'a généralement pas été remarqué dans d'autres travaux sur à peine:

6) Peu à peu, bizarrement, la soif disparut. Je me sentais de plus en plus faible, mais je n'avais plus soif. À peine faim. Nous continuions à parler tous les deux avec Kelmar. (Claudel, Le rapport de Brodeck, 2007)

c) Il faut ajouter que, lorsque $X$ est un verbe fini, ce segment précède toujours $A p_{1}$, la structure étant $X$ à peine, comme on pouvait le remarquer en (3) ou dans les exemples suivants:

3) Mais cette histoire ne finit pas, elle commence à peine. (Nothomb, Hygiène de l'assassin, 2004)

7) La porte s'est ouverte à peine, elle aussi sans bruit. (Claudel, Le rapport de Brodeck, 2007)

8) Il sait à peine euh écrire euh même ni compter mais c'est pas un gamin qui va passer un CAP pour avoir un métier parce que pour passer un CAP il faut quand même être calé [ESLO1_ENT_077] ${ }^{4}$

d) Enfin, il est possible mais rare que $A p_{1}$ porte sur un adverbe en -ment comme en 9):

4 Les occurrences orales sont toutes extraites du corpus ESLO (Enquêtes sociolinguistiques à Orléans). http://eslo.huma-num.fr/index.php?option=com_ content\&view=article\&id $=1$ 
9) Il a été décoré en 2008 par Nicolas Sarkozy et est régulièrement missionné (à peine officieusement) par Claude Guéant, le secrétaire général de l'Élysée, notamment dans cette république pétrolière qu'est le Gabon. (Legrand, Ce n'est rien qu'un président qui nous fait perdre du temps, 2010)

e) $A p_{1}$ peut également être suivi ou précédé d'un groupe nominal à valeur quantitative:

10) La Terre a énormément changé en à peine 15 ans: regardez les GIF de la Nasa. (http://www.slate.fr/, 26/12/2014)

11) À 16 ans à peine, Damien Galantest un génie de l'informatique. (www.rtl.be/, 6/08/2015)

12) Les architectes de Bayazid ont réalisé la maquette en trois jours à peine, et elle trône maintenant sur un riche présentoir, ce qui irrite l'artiste. (Énard, Parle-leur de batailles, de rois et d'éléphants, 2010).

13) L1: Elle a commencé à aller mieux elle avait bien une dizaine d'années

L2: hm hm

L1: Elle avait été opérée elle avait à peine quatre ans [ESLO1_ENT_101]

f) $A p_{1}$ peut apparaître en incise, ce qui n'est pas le cas pour $A p_{2}$ :

14) Je suis sorti un instant du chapiteau. Trois cents excités, à vue de nez. Moitié moins de CRS en face, l'effectif d'une compagnie, à peine. (Leroy, Le bloc, 2011)

g) $A p_{1}$ peut devenir focus d'une phrase seulement quand il est précédé d'une forme verbale, ce qui exclut cette propriété chez $A p_{2}$ :

3a) C'est à peine que cette histoire a commencée.

7a) C'est à peine que la porte s'est ouverte.

8a) C'est à peine qu'il sait écrire.

h) Lorsque $X$ est une forme verbale, $A p_{1}$ peut se présenter aussi sous la forme c'est à peine si, et dans ce cas il se situe en tête de l'énoncé et porte sur le verbe. On peut donc considérer c'est à peine si comme une variante de $A p_{1}$ devant un constituant verbal:

15) 16 décembre. [...] Lorsque la cloche sonne, tout le monde est pressé de se quitter. C'est à peine si on se souhaite de bonnes fêtes. (http://www.la-croix.com, 21/12/2010) 
16) Hier on se regardait à peine

C'est à peine si l'on se penchait

Aujourd'hui nos regards sont suspendus (Bashung, Résidents de la République, 2008)

17) Ont-ils obtenu quoi que ce soit? Leur cause est-elle un tant soit peu populaire? C'est à peine si on leur consacre un entrefilet dans les journaux nationaux. (Énard, Bréviaire des artificiers, 2007)

On pourrait considérer qu'il s'agit tout simplement d'un procédé emphatique, vu que, apparemment, la suppression d'un des éléments ne change rien au sens de la construction en général; en fait, en absence de verbe le si disparaît:

18) Avouez que c'est cauchemardesque: être rejetée par un groupe, s'en rendre compte et ne pas bouger un doigt pour empêcher cette exclusion! C'est à peine humain 5 ... Je me demande si elle a un cœur, cette Olga. (Schmitt, Odette Toulemonde et autres histoires, 2006)

Et dans tous les cas, on peut transformer la phrase, en ne laissant que le marqueur tout seul, ce qui prouve qu'il s'agit de $A p_{1}$ :

16a) C'est à peine si l'on se penchait.

16b) À peine si l'on se penchait.

16c) On se penchait à peine.

i) $A p_{1}$ peut fournir une réponse absolue à une phrase interrogative ou même former, à lui seul, une interrogation; cette caractéristique, pour des raisons syntaxiques évidentes, n'est pas présente chez $A p_{2}$ :

19) - Ils ont analysé les pelures de crayon?

- Un ami a différé leur arrivée au labo. Il a eu une fièvre.

- Ça te donne quoi? Trois jours de plus?

- À peine. (Vargas, Un lieu incertain, 2010)

20) - Et Harry Quebert? Vous le connaissez bien?

- Non. À peine. Je l'ai croisé quelques fois.

- À peine? m'étonnai-je. Pourtant vous habitez la même ville depuis trente ans. (Dicker, La vérité sur l'affaire Harry Quebert, 2012)

5 Comme le signale un des évaluateurs anonymes, cet exemple pourrait se paraphraser par: «c'est à peine si c'est humain». 


\subsubsection{Propriétés sémantiques}

a) $A p_{1}$, du moins apparemment, affecte un seul élément $p$, sa structure sémantique sera donc du type $A p_{1} p$, alors que $A p_{2}$ construit une relation sémantique entre deux éléments $p$ et $q$.

b) $A p_{1}$ exprime toujours un degré faible dans une échelle sémantique, si bien qu'il a été comparé à d'autres adverbes comme peu ou presque pas avec lesquels il peut commuter.

En revanche, si nous essayons de remplacer $A p_{1}$ par d'autres termes de l'échelle orientés vers un haut degré, les énoncés en question ne sont plus acceptables ou, s'ils le sont, changent complètement leur signification, comme en (20a):

1a) Un au revoir marmonné, à peine (presque pas/??assez/??très) audible.

2a) Pas une glissade, à peine (presque pas) une petite colère passagère.

6a) Mais je n'avais plus soif, à peine (presque pas/??très) faim.

20a) Vous le connaissez bien? - À peine (peu/presque pas/ ??assez/?beaucoup.)

Si on analyse, par exemple (1a), on remarque que la difficulté vient du rapport énonciatif entre "marmonné» et «audible», l'énonciation d'un degré supérieur de "audible» serait incompatible avec l'énonciation de «marmonné». De même, dans l'exemple suivant (21), «nuit noire» serait incompatible avec l'énonciation d'un degré supérieur de «deviner» (tout comme "la nuit est claire» serait incompatible avec «on se devine à peine» et même avec «on se devine» tout court):

21) La nuit est noire, on se devine à peine. (Lemaitre, Au revoir là-haut, 2013)

Cette constatation nous conduit vers deux autres propriétés:

c) la structure sémantique de $A p_{1}$ ne contient pas que $p$ mais aussi $q$ qui, souvent, reste implicite: dans les exemples précédents $p$ est représenté en surface par «audible» et "deviner», respectivement, et $q$ est représenté par "marmonné» et «nuit noire».

d) $A p_{1}$ exige que $p$ et $q$ soient dans une relation sémantique de contradiction. Dans le cas de marmonné/audible la contradiction apparaît explicitement (ce qui est "marmonné» est en principe «non audible»), mais dans le cas de nuit noire/deviner la contradiction s'explique indirectement: «il fait nuit», donc «il faut deviner». 
Il faut donc modifier la structure sémantique de $A p_{1}$ qui serait plutôt de forme $A p_{1} p q$ (plus exactement, comme on le verra, non-q).

Tout cela a été expliqué en vertu d'une certaine insuffisance comparant le fonctionnement de $A p_{1}$ avec celui de $p e u^{6}$. Mais c'est là qu'intervient un nouvel élément qui reste implicite, sous la forme d'un savoir partagé qui établit qu'il faut un degré $x$ de $p$ pour avoir $q$ ( $p x$ donc $q): A p_{1} p$ signifie que le degré de $p$ considéré est inférieur à $x$, disons $x_{-1}$, et donc l'énonciation de $q$ apparaît comme inacceptable: dans l'exemple (1) - marmonné/audible - ce que signale à peine est que «au revoir» $(q)$ est énoncé avec une intensité de la voix $\left(p x_{-1}\right)$ insuffisante pour être "audible» $(x=$ degré de «audible»), et donc comme si «au revoir » n'était pas énoncé, ce qui peut être formalisé par $p x_{-1}$ donc non- $q$.

Cela détermine que dans $A p_{1} p$, $p$ est un prédicat gradable, ce qui ne sera pas le cas pour $A p_{2}, q$ ne l'étant pas nécessairement.

e) La combinaison de $A p_{1}$ avec un syntagme exprimant la quantification (numéral ou indéfini en général) a conduit de nombreux grammairiens et linguistes à y voir un autre sens de Ap. En effet, la commutation laisse d'autres éléments, moins proches de l'orientation négative et plus proches, en revanche, de la restriction (seulement, tout juste) $)^{7}$.

Néanmoins, à notre avis et à cause de la propriété précédente, ces arguments ne sont pas suffisants pour en faire un cas à part. Ce n'est pas la quantité énoncée qui détermine l'insuffisance. Par exemple, $10 €$ est une quantité bien plus petite que $10000 €$, mais à peine est compatible avec les deux:

22) Cela coûte à peine $10 € /$ Cela coûte à peine $10000 €$.

Mais l'incompatibilité se produit lorsque, dans l'énoncé contenant à peine, tant $p$ que $q$ sont explicites:

22a) Cela coûte à peine $10 €$, mon enfant de dix ans pourra l'acheter.

22b) ??Cela coûte à peine $10000 €$, mon enfant de dix ans pourra l'acheter.

6 Voir surtout Ducrot (1991).

7 Cf. Bat-Zeev Shyldkrot (2010, 2012). 
L'explication est donc celle que l'on a proposée plus haut: c'est la «norme» présentée comme partagée par la communauté, et selon laquelle il faut un degré $x$ de $p$ pour avoir $q$.

f) $A p_{1}$ a été aussi qualifié comme un élément proche des termes à polarité négative (TPN), notamment par Bat-Zeev Shyldkrot (2012), dans des contextes où elle exprime une «quasi-négation»: Dans un premier temps on le remarque à peine. Bosque (1980) précise ce caractère en analysant son équivalent espagnol apenas ${ }^{8}$. Mais, tandis qu'en espagnol cette propriété ne l'empêche pas de se combiner avec d'autres adverbes négatifs, en français cela n'est pas possible: à peine rien $^{\star}$ à peine personne ${ }^{\star} \grave{a}$ peine jamais ${ }^{\star}$ sans à peine ${ }^{9}$.

Cette contrainte exige l'emploi d'autres constructions pour rendre une signification plus ou moins analogue à celle de $A p_{1}$. Voici quelques exemples d'énoncés de ce type:

23) Il ne reste quasiment rien de l'hôpital de Kunduz, en Afghanistan. (http://www.sudinfo.be/, 04/10/2015)

23a) Il ne reste/quasiment rien/pratiquement rien/presque plus rien / ${ }^{*} \dot{a}$ peine rien.

24) Comment ce philosophe peut soutenir une pareille thèse, à laquelle pratiquement plus personne ne croit? (Godin, Le Bac Philosophie pour les nuls, 2015)

24a) [...] à laquelle presque personne/peu de personnes croient $/ * a ̀$ peine personne ne croit

25) Les trois marchés ont évolué séparément, sans pratiquement jamais se croiser. (http://www.boilet.net/fr/ nouvellemanga_2006.html/, 12/08/2007)

25a) [...] sans presque jamais se croiser $/{ }^{*}$ sans à peine jamais se croiser.

Ce phénomène met en relief une nouvelle propriété: $A p_{1}$ présente $p$ comme présupposé: «il y a $p$ », ce qui peut être prouvé par un test qui fait intervenir mais:

21a) La nuit est noire, on se devine à peine, mais on se devine.

8 Plus concrètement, Bosque le range parmi les "activateurs négatifs», concept équivalent des «contextes à polarité négative» $(\mathrm{CPN})$, notion décrite en détail par Muller (1991).

9 En espagnol (apenas), cette contrainte n'existe pas et les séquences apenas nada, apenas nadie, apenas nunca, sin apenas sont complètement courantes dans cette langue dans toute sorte de discours. 
18a) C'est à peine humain, mais c'est humain.

Par contre, quasiment $p$ ou presque $p$, etc. ne présupposent pas $p$, mais posent non- $p$. On remarque facilement cette différence dans l'exemple (18):

18b) C'est à peine humain = c'est humain mais à peine

18c) C'est presque humain $={ }^{*}$ 'est humain mais presque

La négation introduirait donc une contradiction avec le présupposé: à peine rien serait interprété comme à peine non-p, ce qui explique que cet opérateur soit incompatible avec la négation.

g) $A p_{1}$ peut se combiner avec mais, et, dans ce cas, la structure est $p$, mais $A p_{1}(p)$, c'est-à-dire $p$ précède $A p$ et celui-ci introduit une précision quant au degré de $p$.

26) Tu aurais dî me gifler quand j'ai dit ça, ou protester, ou t'énerver, mais non, tu as juste tenté de t'expliquer, vexé, mais à peine, non, non, tu te trompes, elle est bonne, généreuse, elle a l'âme élevée. (Lévy, Rien de grave, 2004)

27) Et comment s'appelait le pays en ce temps-là? La Gaule. Eh bien, les voilà enfin les ancêtres qu'il nous fallait. Le retour du héros oublié. Je simplifie, mais à peine. (Reynaert, Nos ancêtres les Gaulois, 2010)

26a) vexé mais /à peine/presque pas?/ peu

27a) Je simplifie, mais [je simplifie]/à peine/presque pas?/peu

En faisant le bilan de l'analyse de cette entité sémantique, $A p_{1}$ présente $p$ comme étant un prédicat gradable et sélectionne un degré de $p$ considéré faible pour rendre acceptable l'énonciation de $q$, et ceci en vertu d'un savoir partagé qui établit un certain rapport de cause entre $p$ et $q$ : il faut un degré $x$ de $p$ pour avoir $q(p x$ donc $q)$.

\section{2. $A p_{2}$}

\subsubsection{Propriétés distributionnelles}

a) La structure de $A p_{2}$ répond généralement au type à peine $X$, (que) $\mathrm{Y}$, où $\mathrm{X}$ et $\mathrm{Y}$ sont des énoncés:

28) À peine arrivé à Saint-Denis, Dieudonné déjà expulsé. (http:// www.leparisien.fr/saint-denis/, 18/09/2015) 
29) le général de Gaulle à peine a-t-il quitté le pouvoir en janvier dix-neuf cent quarante-six qu'il se lance dans une sorte de campagne à très très long cours. [ESLO2_CONF_1241]

b) L'on peut néanmoins trouver la structure inverse: $Y$, à peine $X$ :

30) Tu t'étais précipité vers lui, à peine Dorgelles avait-il levé la séance. Tu l'avais coincé entre quatre yeux. (Leroy, Le bloc, 2011)

c) Le lien entre $X$ et $Y$ présente de nombreuses variantes. D'abord, les deux segments phrastiques peuvent être juxtaposés, comme dans l'exemple (4):

4) À peine la frégate amarrée, le sculpteur a sauté à terre.

Ou bien ils sont liés par le coordonnant $e t$, souvent suivi d'un adverbe temporel, tel que déjà:

31) [...] CHLOE: Il est vraiment pas mal, je trouve. À mon avis, c'est un Scorpion. Et comme vous êtes Poissons, c'est idéal. NATHALIE: Je l'ai à peine vu et vous faites déjà des prévisions? (Foenkinos, La délicatesse, 2009)

32) Pour la plupart des commerçants, la saison a été difficile. "Avec le mauvais temps qu'on a eu, sans vraiment de printemps, je viens à peine de commencer à vendre des chemisettes ou des polos, et déjà, je dois les mettre en soldes». (Libération, 26/06/2013)

Le segment $Y$ est aussi introduit par que - le subordonnant le plus employé - soit par d'autres conjonctions à valeur temporelle, comme quand ou lorsque:

33) euh à peine la messe était finie que tout le monde se précipite vers la sortie [ESLO1_INTPERS_417]

34) Hélène m'invita à déjeuner. J'acceptai. Nous avions à peine fini de manger que quelqu'un sonna. (Némirowsky, Chaleur du sang, 2007)

35) Dans le couloir, Michaël retrouvait à peine sa respiration quand il entendit la voix grave le rappeler. (Lévy, Sept jours pour une éternité..., 2002)

36) L'aube se levait à peine lorsque j'étais sortie du taxi, avais tendu mon bagage au portier. (Djian, "Oh... », 2012).

Enfin, (37) est du même type, mais le verbe de $X$ y est élidé: «à peine cinq minutes s'étaient écoulés...»: 
37) Le serveur empressé déposait sur le Formica un peu gluant des verres et une carafe d'eau glacée. Ma mère commandait dans un arabe parfait. À peine cinq minutes et les plats arrivaient sur la table. (Barbery, Une gourmandise, 2000)

d) Contrairement à $A p_{1}, A p_{2}$ n'est pas le focus d'une phrase, mais admet d'autres procédés d'emphase:

38) Ce malheur m'avait fait penser à François Mitterand. Il avait passé sa vie à batailler comme un acharné pour devenir président de la République, et voilà qu’à peine élu on lui annonçait un cancer. (Foenkinos, Je vais mieux, 2014)

\subsubsection{Propriétés sémantiques}

a) $A p_{2}$ construit une relation sémantique entre deux éléments $p$ et $q$; sa structure sémantique serait donc de la forme $A p_{2} p, q$, $p$ et $q$ étant par ailleurs des énoncés, contrairement à ce qui est propre à $A p_{1}$.

b) La caractéristique apparemment plus évidente de $A p_{2}$ du point de vue sémantique est que cet opérateur met en relation deux procès où l'un est immédiatement postérieur à l'autre, $A p_{2}$ marquant celui qui est temporellement antérieur. Il signale la succession immédiate entre deux actions, insistant sur la brièveté de l'intervalle de temps qui s'y est écoulé. La présence d'autres adverbes de temps dans $q$ ou l'emploi de quelques structures à valeur emphatique renforcent cet effet:

32a) Je viens à peine de commencer à vendre des chemisettes ou des polos, et déjà je dois les mettre en soldes.

35a) Dans le couloir, Michaël retrouvait à peine sa respiration quand il entendit la voix grave le rappeler.

36a) L'aube se levait à peine lorsque j'étais sortie du taxi.

Ou bien d'autres procédés sont employés pour chercher le même effet:

38a) Voilà qu'à peine élu on lui annonçait un cancer.

Étant donné que le segment $Y$ se présente normalement comme immédiatement postérieur à $X$, il est assez exceptionnel qu'il soit introduit par la locution exprimant l'antériorité temporelle comme avant que $e^{10}$ :

10 Faute d'espace, nous ne développerons pas cette affirmation à propos du rapport entre à peine et avant que. 
39) Les cloches de grace Cathedral sonnèrent et Zofia et Lucas eurent à peine le temps de se faufiler dans l'étroit corridor avant que toutes les portes du grand hall ne se referment. (Lévy, Sept jours pour une éternité..., 2002)

c) De même que, dans le cas de $A p_{1}$, la comparaison avec d'autres marqueurs (quasiment, presque) nous a conduit à considérer que $A p_{1}$ $p$ présuppose $p$, dans le cas de $A p_{2}$, la comparaison avec aussitôt (que), un marqueur exprimant plus nettement la successivité temporelle, montre que la relation entre $p$ et $q$ n'est pas d'ordre temporel mais plutôt d'ordre aspectuel: $A p_{2}$ signale que c'est tout au début de $p$ que $q$ survient, $A p_{2}$ signale la borne initiale du procès $p$.

On peut ainsi voir que $A p_{2} p$ admet la paraphrase commencer $\grave{a} p$, d'ailleurs le verbe commencer apparaît fréquemment dans le contexte de $A p_{2}{ }^{11}$, ainsi que la forme verbale venir $d e^{12}$ :

35b) Dans le couloir, Michaël retrouvait/commençait à retrouver/ venait de retrouver/à peine sa respiration quand il entendit la voix grave le rappeler.

36b) L'aube se levait à peine /commençait à se lever/venait de se lever/lorsque j'étais sortie du taxi.

40) Elle avait à peine achevé/venait d'achever sa phrase qu'un immense éclair diffusa dans la brume un halo de lumière fulgurant ${ }^{13}$. (Lévy, Sept jours pour une éternité..., 2002)

d) Dans le cas de $A p_{2}$, il n'y a apparemment pas de contrainte qui détermine la relation énonciative entre $p$ et $q$, comme c'est le cas pour $A p_{1}$ (relation de contradiction entre $p$ et $q$, comme on l'a vu): quelle relation existerait-il entre «l'aube se lever» et «sortir du taxi»(36), ou entre "achever sa phrase» et "un halo de lumière» (40)? En effet, la relation entre $p$ et $q$ ne préexiste pas à l'énonciation, autrement dit, elle n'est pas linguistique (dans le sens qu'elle n'est pas dans la langue), mais énonciative, et pourtant le locuteur la présente comme si elle était effectivement préalable, comme une «norme» implicite, admise par

11 Cette remarque reste valable pour $A p_{1}$ quand celui-ci porte sur un verbe.

12 Le rapprochement entre «à peine temporel» (notre $A p_{2}$ ) et le passé récent venir de a été aussi fait par Bat-Zeev Shyldkrot (2012: 93).

13 En (40) la substitution par la structure commencer à est impossible à cause du lexème verbal d'origine («achever»). 
la communauté linguistique ${ }^{14}$. En effet, dans l'exemple (36), l'énoncé présente «sortir du taxi» comme entretenant une relation énonciative avec «l'aube levée» (on prend un taxi quand l'aube est levée).

e) $A p_{2}$ opère justement sur cette relation entre $p$ et $q$, pour signaler que la situation actuelle contrevient à ce qui est admis par la communauté linguistique. C'est ainsi que l'énoncé contenant $A p_{2}$ admet l'enchaînement avec «contrairement à ce qui est habituel», ou une expression équivalente:

28a) À peine arrivé à Saint-Denis, Dieudonné déjà expulsé, contrairement à ce qui est habituel/normal.

33a) À peine la messe était finie que tout le monde se précipite vers la sortie, contrairement à ce qui est habituel/normal. ${ }^{15}$

Ainsi, dans (28), le locuteur implicitement rappelle que normalement on n'est pas expulsé juste au moment d'arriver dans un endroit, et dans (33) on sait que, habituellement, on sort de la messe sans précipitation.

f) $A p_{2}$, tout comme $A p_{1}$, peut se combiner avec mais, cette fois-ci sous la forme mais $A p_{2} p, q$. Dans ce cas mais reste extérieur à $A p_{2} p q$, établissant la connexion avec un énoncé précédent; au contraire, avec $A p_{1}$, mais affecte seulement $p$ :

41) Mon père était timide... Ses lettres étaient affectueuses [...]; mais à peine étions-nous en présence l'un de l'autre, qu'il $y$ avait en lui quelque chose de contraint que je ne pouvais m'expliquer, et qui réagissait sur moi de manière pénible. (Pennac, Chagrin d'école, 2007)

Enfin, comme on l'a fait pour $A p_{1}$, nous pouvons synthétiser la signification de $A p_{2}$. Cette entité sémantique présente $p$ et $q$ comme entretenant une certaine relation sémantique qui est admise par la communauté linguistique et présente l'énonciation de $p$ comme ne légitimant pas totalement l'énonciation de $q . A p_{2} p, q$ se révèle ainsi comme une exception à une norme.

14 Il s'agit d'une notion proche du stéréotype tel qu'il est défini chez Anscombre (2001); néanmoins, dans notre cas, nous préférons plutôt parler de norme.

15 Il faut préciser que la paraphrase serait plutôt: "contrairement à ce que $X / O N$ présente comme habituel/ normal». 
JESÚS VÁZQUEZ MOLINA

\section{Conclusion}

Nous avons essayé de montrer que l'analyse de à peine (Ap) comme opérateur discursif décrit au moyen d'une série de propriétés formelles et sémantico-pragmatiques, permet de faire ressortir à la fois les traits communs entre ses divers emplois et leurs différences distributionnelles et sémantiques. En effet, l'hypothèse initiale, basée sur l'observation du corpus étudié, permettait d'y voir certaines significations différentes, et en cela nous accordons avec la majorité des analyses préalables sur à peine. Ensuite, l'analyse détaillée nous a fait retenir deux valeurs uniques de l'opérateur $\left(A p_{1}\right.$ et $\left.A p_{2}\right)$. Les deux entités sémantiques ont en commun l'évocation d'une «norme»; dans le cas de $A p_{1}$, cette norme fait intervenir la gradualité, ce qui n'est pas le cas pour $A p_{2}$. Dans ce dernier cas, c'est l'exception à cette norme généralement évoquée qui devient le trait fondamental pour la description de $A p_{2}$.

\section{Bibliographie}

ANSCOMBRE J.-C. (2001), Le rôle du lexique dans la théorie des stéréotypes, Langages 142, 57-76.

ANSCOMBRE J.-C., DONAIRE, M.-L. \& HAILLET, P. P. (éds) (2013), Opérateurs discursifs du français, Berne, Peter Lang.

BAT-ZEEV SHYLDKROT H. (2010), Polysémie de à peine, L'information grammaticale 125, 12-16.

BAT-ZEEV SHYLDKROT H. (2012), Concurrence et substitution: le cas de à peine et difficilement, in Berlan F. \& Berthomieu G. (dir.), La synonymie, Paris, PUPS, 91-102.

BOSQUE I. (1980), Sobre la negación, Madrid, Cátedra.

DUCROT O. (1991 [1972]), Dire et ne pas dire, Paris, Hermann.

GREVISSE M. \& GOOSSE A. (2011), Le bon usage, 15e éd., Bruxelles, De Boeck-Duculot.

HANSE J. \& BLAMPAIN D. (2012), Dictionnaire des difficultés du français, 6e éd., Bruxelles, De Boeck-Duculot.

JAYEZ J. (1987), Sémantique et approximation: le cas de presque et $\grave{a}$ peine, Lingvisticae Investigationes 11: 1, 157-196.

LEEMAN D. (2004), L'emploi de juste comme adverbe d'énonciation, Langue Française 142, 17-30.

MELLET S. \& MONTE M. (2009), Juste / à peine et la construction de la frontière notionnelle, Cahiers de Praxématique 53, 33-56.

MULLER C. (1991), La négation en français, Genève, Droz. 
REY-DEBOVE J. \& REY A. (éds), (2011), Le Petit Robert 2011, Editions Le Robert.

ROUANNE L. (1998), Les différentes portées de presque et de à peine en fonction de l'aspect lexical des actions modifiées, Thélème 13, 185-195.

SANDFELD K. (1965), Syntaxe du français contemporain, vol.2, Paris, Honoré Champion.

VÁZQUEZ MOLINA J. (à paraître), La complexité sémantique d'un marqueur discursif: à peine, Actes du colloque RSL VI, Nantes, 2013.

Dictionnaire de l'Académie française, $9^{e}$ éd: http://atilf.atilf.fr/academie 9.htm

Trésor de la langue française informatisé: http://atilf.atilf.fr/

Corpus ESLO: http://eslo.huma-num.fr/index.php?option=com content\&view $=$ article\&id $=1$ 